\title{
A "smart" trap device for detection of crawling insects and other arthropods in urban environments.
}

Panagiotis Eliopoulos ${ }^{1}$, Nikolaos-Alexandros Tatlas ${ }^{2}$, Iraklis Rigakis ${ }^{3}$, Ilyas Potamitis ${ }^{2 *}$

1 Dept. of Agriculture Technologists, Technological Educational Institute of Thessaly, Larissa 41110, Greece; eliopoulos@teilar.gr

2 Department of Electronics Engineering, Piraeus University of Applied Sciences, Athens 12244, Athens, Greece; ntatlas@puas.gr

3 Dept. of Electronics, Technological Educational Institute of Crete, Chania, 73133, Greece; rigakis@chania.teicrete.gr

* Author to whom correspondence should be addressed; E-Mail: potamitis@staff.teicrete.gr; Tel.: +30 28310 21900; Fax: +30 2831021913

\begin{abstract}
We introduce a device for automatic detection and reporting of crawling insects in urban environments. It is a monitoring device for urban pests that complies with the context of smart homes, smart cities and is compatible with the emerging discipline of the Internet of Things (IoT). We believe it can find its place to every room of a hotel, hospital, military camp and residence. This box-shaped device attracts targeted insect pests, senses the entering insect and takes automatically a picture of the internal space of the box. The picture is communicated through the Wi-Fi commonly found in such establishments to an authorized person/stakeholder receiving the picture to take proper action. The e-trap includes strong attractants (pheromone and/or food) to increase capture efficiency. The insect is trapped on the sticky floor of the device. The device carries the necessary optoelectronic sensors to guard all entrances of the trap. As the insect enters it interrupts the infrared light source. This triggers a detection event; a picture is taken, and a time-stamp is set before reporting the event through the $\mathrm{Wi}-\mathrm{Fi}$. The device can be integrated seamlessly in urban environments and operates unobtrusively to human activities. We report results on various insect pests and depending on the insect species, can reach a detection accuracy ranging from 96$99 \%$.
\end{abstract}




\section{Introduction}

The quality of life for most people in the future will be represented in the cities' quality of life index. The European Commission has, in recent years, been increasing its focus on urban issues, as a response to the fact that by 2020 it is estimated that almost $80 \%$ of EU citizens will be living in cities [1]. Cities provide development and growth, and generally a better life than in rural areas. Apart from quality of living, houses, buildings, roads, schools, hospitals, markets and other features, that characterize the urban environment, provide habitats for a special group of insects and other arthropods, some of which attained pest status, the so-called "urban insects" [24].

Contrarily to agroecosystems, pest status in the urban environment may not be based on a measurable feature. The damage and the control treatments costs cannot always be determined. Structural damage, sanitary problems, health threats, or simple annoyance only by the insect presence, have been developed to common cases in urban environments. A decision to apply control measures maybe based on potential damage or personal injury, or solely or in part on emotion. Arthropods in the urban environment are completely unacceptable, whether their populations are low or high. Pest management and control strategies are based on early detection and pest identification before application of effective chemical and nonchemical control treatments.

Early pest detection is crucial for effective and affordable control in urban environments. For this purpose, various trap types (mainly sticky) are frequently used by homeowners, pest management professionals and urban entomologist researchers for detecting infestations of crawling insects like ants, termites, bedbugs, cockroaches, earwigs, beetles, moth larvae etc. as well as other crawling arthropods such as spiders, millipedes, centipedes etc $[2,5,6]$. These devices provide consistent estimates not only of insects' presence (detection) and relative abundance (monitoring) but they are also useful for evaluating insecticide treatment efficacy (post-treatment analysis) and control purposes (population reduction) [7-9].

As a detection tool, sticky traps provide information on distribution and population density, thereby assisting in properly targeting insecticide applications. Because of their safety, ease of use, and non-toxicity, sticky traps are considered to be a valuable tool in integrated pest management (IPM) programs especially in urban environments.

For the purposes of this study, trapping tests were performed for the detection of three very common crawling insects in urban environments. 
A. Cockroaches (order Blattodea). They are cosmopolitan insects, occurring in nearly all over the world. Although, most of the approximately 4000 described species live in small populations in forest habitats, their association with decaying organic matter and humid conditions maintains some species (nearly 30 ) in the urban environment. They may become very serious pests given that they can passively transport pathogenic microbes on their body surfaces, particularly in environments such as hospitals $[10,11]$, they are often linked with allergic reactions in humans [12] and asthma [13]. These allergens are heat-stable and persistent in the living space. Approximately $20-50 \%$ of homes with no visible sign of cockroaches have detectable cockroach allergens in dust [14].

B. Ants (Hymenoptera: Formicidae). The pest status of ants is based on their nesting and foraging habits. The urban environment provides a variety of soil types and conditions, suitable for a large number of ant species [2, 15]. Nesting along building foundations can result in damage to structural members of buildings, and to exterior faces. Ants usually enter the buildings to find food, demonstrating remarkable persistence foraging inside and establishing satellite nests indoors. The habit of foraging ants to visit a variety of food sources increases their potential to acquire pathogenic and food decay microbes. A large number of pathogens have been recorded from ant species, including bacteria (Burkholderia, Clostridium, Enterobacter, Salmonella etc) and fungi (Aspergillus, Penicillium etc), $[2,15,16]$. Pest ants in the urban environment have a significant economic impact both on the pest control industry and the general public. Many times, ants have been ranked as the number one pest problem of households, even surpassing cockroaches [17]. Most important and well studied species are the fire ants (Solenopsis sp.), because of their medical and agricultural impact [18]; the Pharaoh ants (Monomorium pharaonis), which are major household pests and can act as disease vectors in hospitals [19]; and carpenter ants (Camponotus spp.), which are important wood-destroying organisms [20].

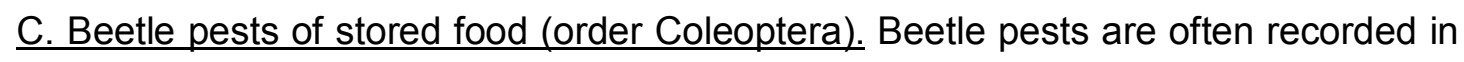
urban environments searching for food (cereals, grains, packaged food, flour etc). Their presence may become a serious problem in certain cases like in residences, markets, hospitals, bakeries, restaurants etc. There is a plethora of beetle species (Sitophilus, Rhyzoperta, Tribolium, Cryptolestes, Oryzaephilus, Stegobium, Lasioderma etc) invading abovementioned urban environments searching for food $[21,22]$.

Recently, we observe an upsurge of interest on shifting sophisticated, high-tech procedures to insect sensing that are normally encountered in other research fields (see the work of Brydegaard in Lidar applications on insect fauna [23], Perles in optical 
sensing of termites [24], Potamitis for electronic e-traps for flying insects [25-28], Zhong on applying deep learning to insects trapped on sticky traps [29]). This study belongs to this general trend but is different to its aim and cause. Our study aims to introduce a novel trap-device that can be hidden conveniently under a bed or fixed to the wall that can become a useful tool for the automated early detection of crawling insects and other arthropods in urban environments. Though we have tested three widely encountered cases the applicability can be directly extended to other cases as well, namely: The presence mainly of Bed Bugs (Hemiptera: Cimicidae) and Cloth Moths that feed on stored fabrics (Lepidoptera: Tineidae) can be devastating for the reputation and prospect of a hotel or hospital in the era of TripAdvisor, Instagram and Facebook. The proposed device will curtail the cost of Integrated Pest Management (IPM) applied to hotels as it will limit the application of IPM from a prescheduled basis (i.e. once a year) to an on-time, localized (i.e. per room) application of IPM. More on, it allows the hotel to advertise that it takes best action to protect its customers and their children by applying surveillance control on insects.

\section{Materials \& Methods}

\section{Trap Design \& Function}

This box-shaped device having the size of a shoe (Fig.1), attracts insect pests, senses the entrance of the insect and takes automatically a picture of the internal space of the box. The picture is communicated through the WiFi commonly found in such establishments to an authorized person/stakeholder receiving the picture to take proper action. In this way, continuous, accurate and real - time detection is achieved, without the need for human intervention. It is a monitoring device for urban pests in the context of smart homes, smart cities and is compatible with the emerging discipline of the Internet of Things (loT) (Fig. 2).
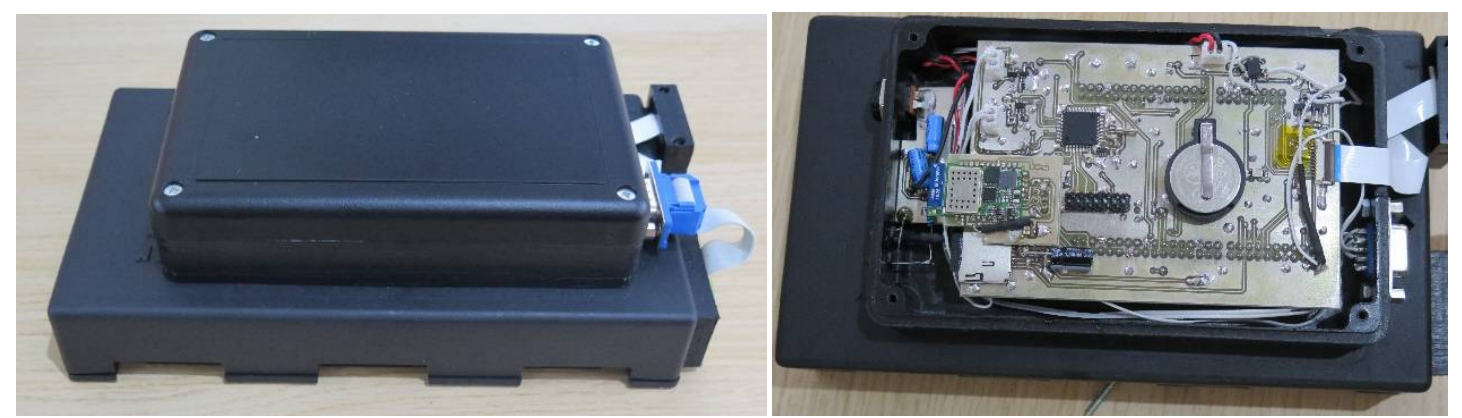

Fig. 1. (Left) A prototype of the smart trap. (Right) The electronics are mounted on top of a plastic trap. 
Our smart trap functions like a classic floor trap. Traps may differ greatly in design features (shape, size, surface material etc) and the presence of attractants (food, pheromone). All these factors along with placement method influence dramatically the trap efficacy [30].

The box includes a strong attractant to attract the insects and maximize captures. The insect is trapped on a sticky surface that is inside the device, basically a cardboard coated with special insect glue (Tanglefoot $\circledast$ Tangle-Trap $®$ Sticky Coatings) that lasts (remains sticky) for 4-6 months. The sticky floor provides the means for immediate verification of reported results. Captured insects release additional pheromone by themselves and increase the attractiveness. The presence of multiple attractants targeting different insect species simultaneously is also possible and recommended.

The concept presented in this work does not depend on a specific trap configuration and many types are compatible with it. In this work, we modified a wellknown trap for crawling insects (TRAPPER Pest Monitor, Bell Labs, US) on which we added the electronic components (see Fig 1-left). This trap has multiple entrances that are all monitored simultaneously by a single laser beam (infrared laser PN: 980MD30-1230-CAB/NANMA). Any entering point will interrupt the laser beam and this triggers a time counter.

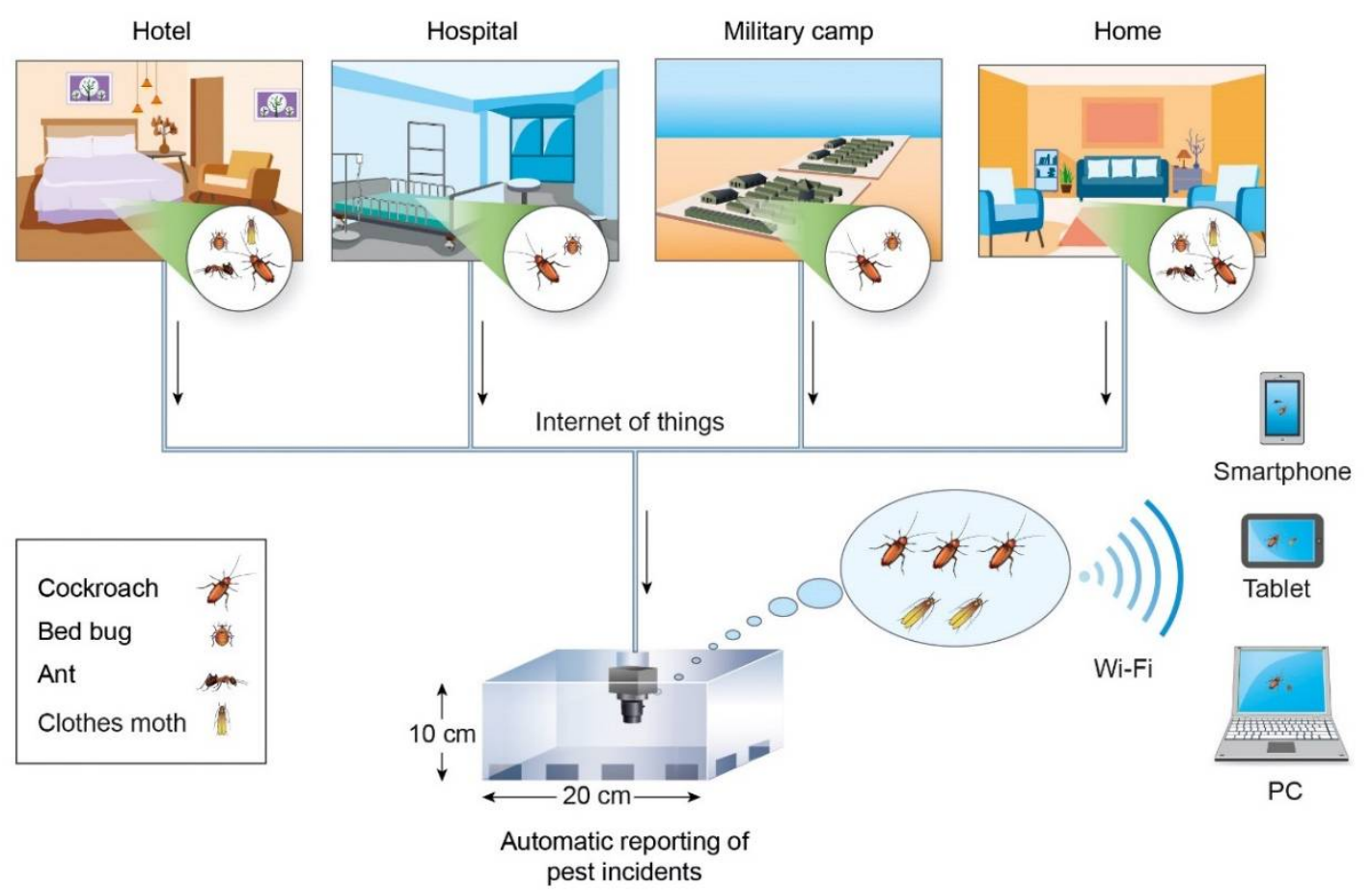

Fig. 2: Surveillance of urban, crawling insects in the context of smart-cities. Residential accounts, food processing plants, military camps and hospitals are some of the main locations that can be monitored independently or as part of a regional/country network in the context of loT. 
After 20 seconds a photograph (Omnivision, Camera OV2640, cmos sensor camera module) of the internal volume of the trap is taken (Fig.3), time-stamped and delivered to prestored mail addresses while a copy of the picture is stored internally in the SD card of the device. The time delay ensures that is given enough time to the insect that follows the chemical signals of the bait to crawl inside. Several time constraints are applied to ensure that the device is not reporting a false alarm. The processor that handles all tasks is a STM32F767 ARM Microcontroller (see Fig 1-right).

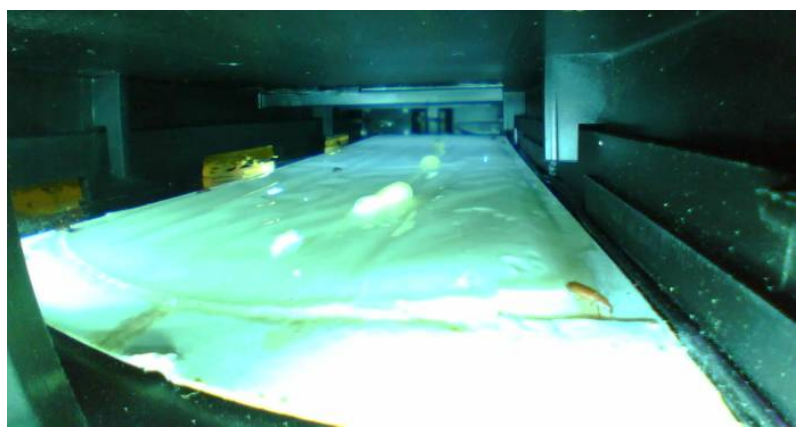

Fig. 3: Photograph from the internal of the trap, automatically taken 20 seconds after insect's entrance (an adult beetle is depicted in the down right corner).

\section{Evaluation}

For the purposes of our study, a prototype of this trap was tested under real conditions in three cases. Our trap was placed:

a) in an old food warehouse with an increased presence of cockroaches in Agrinio, Central Greece. Trap placement lasted from early to mid May 2018.

b) in a livestock unit with large ant populations in Larissa, Central Greece. Trap placement lasted from early to mid April 2018.

c) in an old-style granary (horizontal type) with a heavy insect infestation in stored wheat mass in Farsala, Central Greece. Trap placement lasted from early to mid March 2018.

Trap placement lasted about two weeks in all cases. The trap was manually checked on a daily basis, and the number of captured insect individuals was recorded. These manual records were compared with the data that were automatically recorded by the trap and sent wirelessly. Non-target insects or other arthropods that may enter the trap accidentally were not included in the results. During each manual inspection the sticky surface with captured insects was removed for examination in the lab (Fig.4) and replaced by an identical one clean sticky cardboard surface. In all cases special attractant was placed in the centre of sticky surface in order to achieve sufficient number of captures. Baits applied for each target were: pure wheat germ oil (HealthAid, 
UK) for stored food beetles, $25 \%$ sugar solution for ants, and Trapper roach attractant (Bell Laboratories, USA) for cockroaches.
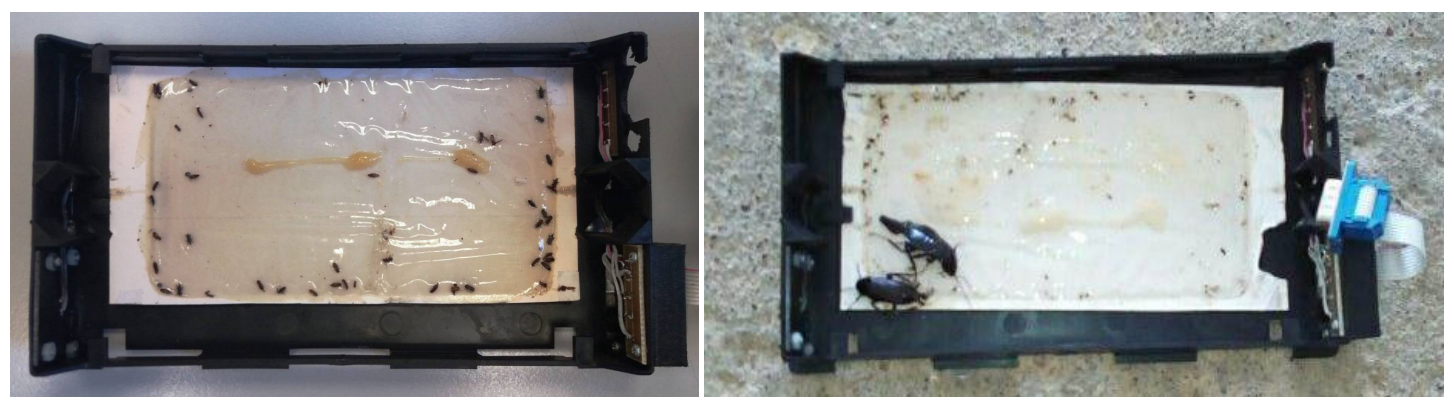

Fig. 4. Evaluation of reported results. Sticky surface inside the trap after 2 days of placement in (left) a cereal grain storage facility with serious insect infestations, (right) in an old food warehouse with an increased presence of cockroaches.

\section{Results \& Discussion}

Results from the evaluation of the prototype trap are presented in Fig 5. As it is clearly depicted, our smart trap is very accurate, achieving 96-99\% accuracy on automatic counts compared with manually counted numbers of captured insects. The accuracy of our system in detecting insect presence is also shown by the very high correlation ( $r>0.96$ in all cases) between the generated signals and actual numbers of insects caught in the trap. 

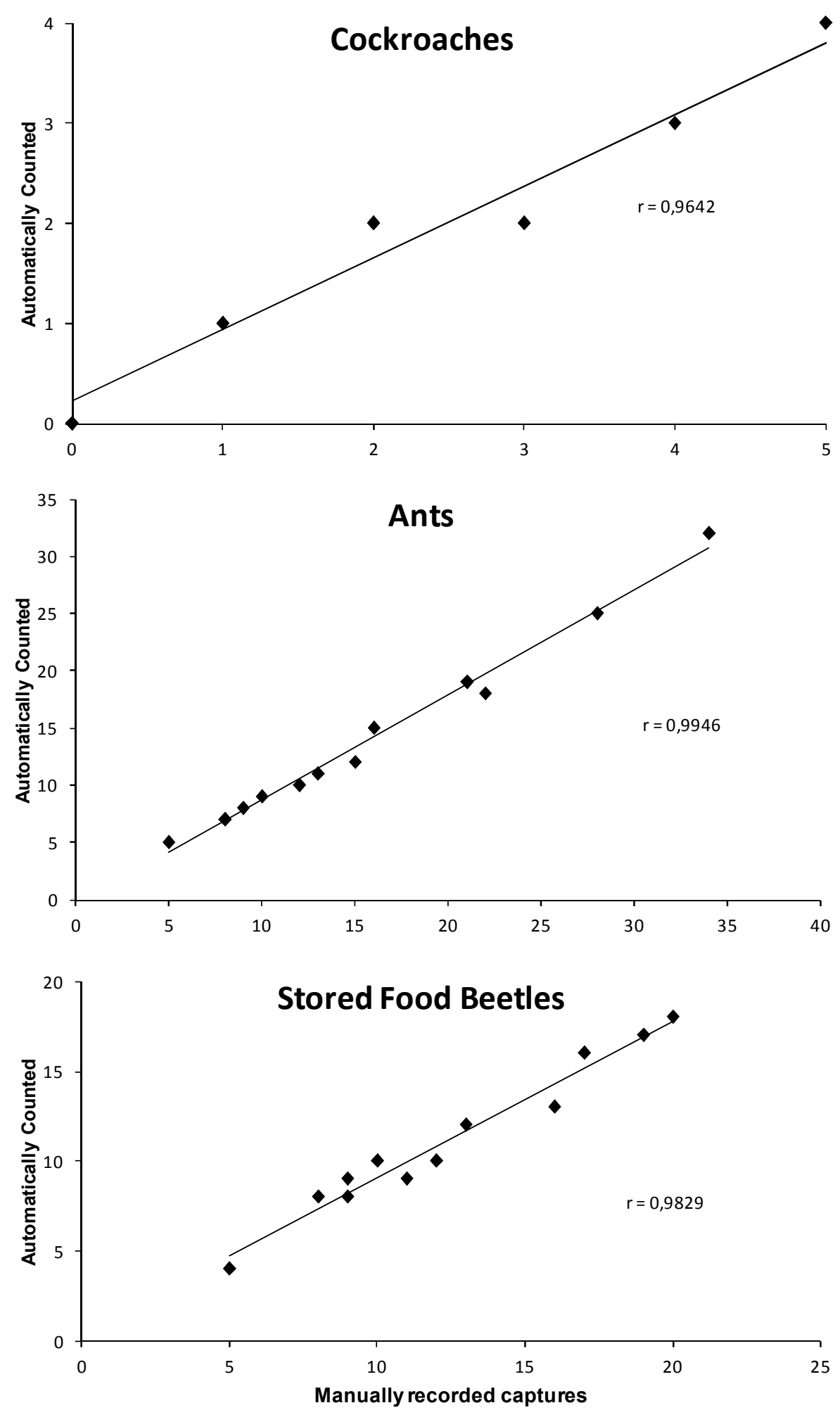

Fig. 5. Accuracy of the automatic counting in comparison with actual detection, each species target insect. The values of the correlation coefficient $r$ prove that our system is $>96 \%$ accurate (when detected and counted values are the same then $r$ equals to 1) 
To the best of our knowledge, it is the first time that an automated remote system is evaluated under real conditions in detecting insect pests in urban environments. A few lab studies have been carried out on the development of similar systems for the detection of urban insects [31, 32]. The accuracy of our system is higher than all of the abovementioned monitoring systems.

Our study aims to introduce a novel trap-device that we believe it can find its place to every urban environment such as houses, hotel rooms, hospitals, military camps and food markets and storages. Such a device will curtail the cost of Integrated Pest Management (IPM) applied to hotels as it will limit the application of IPM from a prescheduled basis (i.e. once a year) to an on-time, localized (i.e. per room) application of IPM. More on, it allows the hotel to advertise that it takes best action to protect its customers and its children by applying surveillance control on insects. Hospitals can be equipped with such a device per room that can be hidden conveniently under a bed or fixed to the wall.

It has been well documented that insects may act as a "pathogen reservoir", which especially in a hospital environment, may cause serious health hazards [33-35]. Insect problems can be detrimental for the financial prospect of a private hospital establishment or for the managemental capability of the head of the hotel and finally of the Ministry of Health [36].

Again, the presence of Ants, Cockroaches, Bed Bugs or stored food insects in home residencies, schools or even in Military Camps may become a serious problem with unforeseen consequences [2,37]. With a cost of a good alarm clock the device has the prospect to be widely accepted by typical residencies at least to one device per house.

The suggested product conforms with the concept of the smart house and through the emerging trend of loT it can connect the concept of a smart house to a smart city that keeps an eye-watch on the insects.

\section{Conclusions}

With the recent advancements in wireless communications, networks and integrated sensors, the ability to use Wireless Sensor Networks (WSNs) to cover large areas with a network of loT nodes that collectively transmit information to a central server in real-time has become available. WSNs have been demonstrated to exhibit the potential of being used in massive scale for environmental parameter monitoring. While they are constantly evolving to the point of a widely used technology, the requirements for an automated trap, including autonomous operation pose practical 
limitations to the use of off-the-shelf solutions, thus leading to the design and development of custom hardware. This is due to the type of sensor (imaging and presence), the application field, the need for low power operation, adaptable size to match the trap, robustness to environmental conditions, the need for spatial coverage and the existence of isolated or remote monitoring spots. The main concept is to hide a small, affordable device for insect surveillance of urban crawling insects and forget its existence until the user receives a picture in his/her mobile.

The smart trap presented in this work was developed following the above requirements, for fully automated operation. The cost and size will allow multiple traps to be installed in possibly concealed locations such as under beds, as instructed by the IPM strategy with a sole technical requirement of sufficient Wi-Fi coverage, which is nowadays taken for granted in most indoor environments.

As the events are relayed via wireless networking, following the loT architecture, these traps may be integrated to any smart-building infrastructure. This may allow the establishment of applications ranging from end-user notification/alerting in order to take appropriate measures, to condition based insect management depending on set business rules. Moreover, as insect infestation may not be localized on a building basis but be widespread, by centralizing the server infrastructure and providing geolocational information for each smart trap, an interactive infestation map may be created, adding the system to the smart city concept. Future work relates detection of urban insects and deep learning as in the paradigm of Zhong [29], where the device automatically counts the insects and reports their identity and Sadegh et all [30] on wild animals.

\section{References}

1. European Commission (2010). Making Our Cities Attractive and Sustainable: How the EU Contributes to Improving the Urban Environment. Publications Office of the European Union

2. Robinson, W. H. (2005). Urban insects and arachnids: a handbook of urban entomology. Cambridge University Press.

3. McIntyre, N. E. (2000). Ecology of urban arthropods: a review and a call to action. Annals of the Entomological Society of America, 93(4), 825-835.

4. Bonnefoy, X., Kampen, H., \& Sweeney, K. (2008). Public health significance of urban pests. World Health Organization.

5. Dhang, P. (Ed.). (2011). Urban pest management: An environmental perspective. CABI.

6. Dhang, P. (Ed.). (2014). Urban Insect Pests: Sustainable Management Strategies. CABI.

7. Owens, J. M., \& Bennett, G. W. (1983). Comparative study of German cockroach (Dictyoptera: Blattellidae) population sampling techniques. Environmental Entomology, 12(4), 1040-1046. 
8. Kaakeh, W., \& Bennett, G. W. (1997). Evaluation of trapping and vacuuming compared with low-impact insecticide tactics for managing German cockroaches in residences. Journal of economic entomology, 90(4), 976-982.

9. Ballard, J. B., \& Gold, R. E. (1983). Field evaluation of two trap designs used for control of German cockroach populations. Journal of the Kansas Entomological Society, 506-510.

10. Rivault, C., Cloarec, A., \& Le Guyader, A. (1993). Bacterial load of cockroaches in relation to urban environment. Epidemiology \& Infection, 110(2), 317-325.

11. Elgderi, R. M., Ghenghesh, K. S., \& Berbash, N. (2006). Carriage by the German cockroach (Blattella germanica) of multiple-antibiotic-resistant bacteria that are potentially pathogenic to humans, in hospitals and households in Tripoli, Libya. Annals of Tropical Medicine \& Parasitology, 100(1), 55-62.

12. Kutrup, B. (2002). Cockroach infestation in some hospitals in Trabzon, Turkey. Turkish Journal of Zoology, 27(1), 73-77.

13. Kang, B., Vellody, D., Homburger, H., \& Yunginger, J. W. (1979). Cockroach cause of allergic asthma: its specificity and immunologic profile. Joumal of Allergy and Clinical Immunology, 63(2), 80-86.

14. Eggleston, P. A., \& Arruda, L. K. (2001). Ecology and elimination of cockroaches and allergens in the home. Journal of Allergy and Clinical Immunology, 107(3), S422-S429.

15. Rust, M. K., \& Su, N. Y. (2012). Managing social insects of urban importance. Annual review of entomology, 57, 355-375.

16. Máximo, H. J., Felizatti, H. L., Ceccato, M., Cintra-Socolowski, P., \& Beretta, A. L. R. Z. (2014). Ants as vectors of pathogenic microorganisms in a hospital in São Paulo county, Brazil. BMC research notes, 7(1), 554.

17. Whitmore, R. W., Kelly, J. E., \& Reading, P. L. (1992). National home and garden pesticide use survey, final report. Volume I: executive summary, results, and recommendations.

18. Jemal, A., \& Hugh-Jones, M. (1993). A review of the red imported fire ant (Solenopsis invicta Buren) and its impacts on plant, animal, and human health. Preventive Veterinary Medicine, 17(1-2), 19-32.

19. Wetterer, J. K. (2010). Worldwide spread of the pharaoh ant, Monomorium pharaonis (Hymenoptera: For-micidae). Myrmecological News 13:115-129

20. Hansen, L. D., Akre, R. D., Wildey, K., \& Robinson, W. (1993). Urban pest management of carpenter ants. In Proceedings of the first International Conference on Urban Pests. (pp. 271-279).

21. Rees, D. P. (2004). Insects of stored products. Csiro Publishing.

22. Subramanyam, B. (Ed.). (1995). Integrated management of insects in stored products. CRC Press.

23. Jansson S., Brydegaard M., Passive $\mathrm{kHz}$ lidar for the quantification of insect activity and dispersal, Animal Biotelemetry, 20186:6, https://doi.org/10.1186/s40317-018-0151-5

24. Perles, A.; Mercado, R.; Capella, J.V.; Serrano, J.J. Ultra-Low Power Optical Sensor for Xylophagous Insect Detection in Wood. Sensors 2016, 16, 1977.

25. Potamitis I, Rigakis I, Fysarakis K (2015) Insect Biometrics: Optoacoustic Signal Processing and Its Applications to Remote Monitoring of McPhail Type Traps. PLoS ONE 10(11): e0140474. doi:10.1371/journal.pone.0140474

26. Potamitis I., Rigakis I., Tatlas N-A., Automated Surveillance of Fruit Flies.Sensors2017,17, 110.

27. Potamitis I., Rigakis I. Large Aperture Optoelectronic Devices to Record and Timestamp Insects' Wingbeats, IEEE Sensors Journal, Volume: 16, Issue: 15, Aug.1, pp. 6053-6061, 2016.

28. Potamitis, I.; Eliopoulos, P.; Rigakis, I. Automated Remote Insect Surveillance at a Global Scale and the Internet of Things. Robotics 2017, 6, 19. 
29. Zhong, Y.; Gao, J.; Lei, Q.; Zhou, Y. A Vision-Based Counting and Recognition System for Flying Insects in Intelligent Agriculture. Sensors 2018, 18, 1489

30. Wang, C., \& Bennett, G. W. (2006). Comparative study of integrated pest management and baiting for German cockroach management in public housing. Journal of economic entomology, 99(3), 879-885.

31. Mankin, R. W., Hodges, R. D., Nagle, H. T., Schal, C., Pereira, R. M., \& Koehler, P. G. (2010). Acoustic indicators for targeted detection of stored product and urban insect pests by inexpensive infrared, acoustic, and vibrational detection of movement. Journal of economic entomology, 103(5), 1636-1646.

32. Oliver-Villanueva, J. V., \& Abián-Pérez, M. A. (2013). Advanced wireless sensors for termite detection in wood constructions. Wood science and technology, 47(2), 269-280.

33. Fakoorziba, M. R., Eghbal, F., Hassanzadeh, J., \& Moemenbellah-Fard, M. D. (2010). Cockroaches (Periplaneta americana and Blattella germanica) as potential vectors of the pathogenic bacteria found in nosocomial infections. Annals of Tropical Medicine \& Parasitology, 104(6), 521-528.

34. Feizhaddad, M. H., Kassiri, H., Sepand, M. R., \& Ghasemi, F. (2012). Bacteriological survey of American cockroaches in hospitals. Middle East $\mathrm{J}$ Sci Res, 12(7), 985-9.

35. Garcia, F. R. M., \& Lise, F. (2013). Ants associated with pathogenic microorganisms in brazilian hospitals: attention to a silent vector. Acta Scientiarum. Health Sciences, 35(1).

36. Owens, K., \& Pesticides, B. (2003). Healthy hospitals controlling pests without harmful pesticides. In Healthy hospitals controlling pests without harmful pesticides. HCWH.

37. Daar, S., Drlik, T., Olkowski, H., \& Olkowski, W. (1997). IPM for Schools: A HowTo Manual. United States Environmental Protection Agency.

38. Mohammad Sadegh Norouzzadeh, Anh Nguyen, Margaret Kosmala, Alexandra Swanson, Meredith S. Palmer, Craig Packer, Jeff Clune (2018). Automatically identifying, counting, and describing wild animals in camera-trap images with deep learning, Proceedings of the National Academy of Sciences, 201719367; DOI:10.1073/pnas.1719367115 